This item was submitted to Loughborough's Research Repository by the author.

Items in Figshare are protected by copyright, with all rights reserved, unless otherwise indicated.

\title{
Exploring the "impact" in Impact sourcing ventures: a sociology of space perspective
}

PLEASE CITE THE PUBLISHED VERSION

\section{PUBLISHER}

Palgrave

\section{VERSION}

AM (Accepted Manuscript)

\section{PUBLISHER STATEMENT}

This work is made available according to the conditions of the Creative Commons Attribution-NonCommercialNoDerivatives 4.0 International (CC BY-NC-ND 4.0) licence. Full details of this licence are available at: https://creativecommons.org/licenses/by-nc-nd/4.0/

\section{LICENCE}

CC BY-NC-ND 4.0

\section{REPOSITORY RECORD}

Sandeep, M.S., and M.N. Ravishankar. 2019. "Exploring the "impact" in Impact Sourcing Ventures: A Sociology of Space Perspective". figshare. https://hdl.handle.net/2134/19346. 


\title{
Exploring the "impact" in Impact sourcing ventures: A sociology of space perspective
}

M.S.Sandeep, UNSW Business School, The University of New South Wales, Australia. M.N.Ravishankar, School of Business and Economics, Loughborough University, UK.

\begin{abstract}
Using qualitative methods this paper explores the lived experience of individuals employed in impact sourcing ventures. In doing so, the paper attempts to understand "impact" from the point of view of beneficiaries. The paper, drawing on Georg Simmel's work on the sociology of space, explores how space influences the lived experience of beneficiaries in ImS ventures. The findings highlight the various strategies adopted by beneficiaries to navigate the dialectical tensions experienced as a result of living and working in the new (ImS workplace) and the old (community) space. The paper also draws attention to the multifaceted nature of impact.
\end{abstract}

Keywords: Impact sourcing, sociology of space, India, case study 


\section{INTRODUCTION}

Impact sourcing is the practice of bringing digitally-enabled outsourcing jobs to marginalized individuals. The idea that $\operatorname{ImS}$ can play a role in providing sustainable livelihoods to marginalised individuals in the global south is steadily gaining credence among academics and practitioners. The claims of ImS ventures of positively impacting lives of marginalized individuals through employment have been supported by recent empirical inquiries (e.g. Heeks and Arun, 2010; Madon and Sharanappa, 2013; Malik, Nicholson and Morgan, 2013; Lacity, Rottman and Carmel, 2014). Researchers have so far adopted Amartya Sen’s “capabilities approach” (Madon and Sharanappa, 2013; Malik et al., 2013) and the "sustainable livelihoods framework" method (Heeks and Arun, 2010) to understand the impact of ImS on individuals and communities.

In this paper we seek to further explore the nature of "impact" by exploring the lived experience of the "beneficiaries" of $\operatorname{ImS}$ ventures, i.e. the $\operatorname{ImS}$ employees. Here, impact is conceptualised as the behavioural and attitudinal changes that ImS employees experience while working in ImS ventures. The paper argues that the creation of a new space, i.e. the ImS workplace in the community, creates an environment ripe for new experiences and learning. The presumption here is that the dialectical tensions experienced by $\operatorname{ImS}$ employees, who are part of the ImS space and the community space, introduces them to challenges in both spaces, and in confronting those challenges, they "experience” impact. In other words, individuals are coerced to develop new practices and capabilities to confront the challenges posed by new social and cultural settings. The resulting social and psychological changes, the paper suggests, has the potential to transform and impact the individual.

In the broader literature on IT and Business Process Outsourcing (IT-BPO) and call-centre literature, there are some studies that explore the relationship between space and individuals. For instance, Barnes (2007) explored the negative impact space can have by exploring the 
relationship between employees and the built environment in a call centre in Australia. She suggests that physical spaces can engender individual and collective resistance and can be a point of employee dissatisfaction. In another study of hot-desking policies of an organization, Hirst (2011) notes how different people adopted different strategies to thrive in their environment. For instance, individuals who got along tended to occupy workstations close to each other. In these examples, individuals and groups responded to and were impacted by externally imposed spatial conditions. In ImS ventures too, external forces are at play. New recruits who are from predominantly patriarchal, orthodox communities are thrown into a relatively more urbane, glamorous world of IT-BPO. The social and cultural demands of the new space (i.e. the ImS workplace) are very different from what they are used to (in the community space). To unravel how individuals experience and navigate spaces, and develop strategies to overcome challenges in ImS ventures we draw on sociology of space literature. More specifically, we borrow ideas from Georg Simmel's essay titled The Sociology of Space (translated and edited version: Frisby and Featherstone, 1997). To understand the social and psychological changes of individuals, we examine what he terms "sociation", i.e. the interaction between individuals through social encounters. Specifically, using the fundamental qualities of spatial forms as outlined by Simmel, we examine how spaces and places influence the process of sociation and impact the individual. Through an understanding of sociation it is possible to analyse how interactions mediated through spaces shape attitudes and behaviours

of individuals. This paper draws on aforementioned sociology of space literatures and analyses data collected through an in-depth interpretive qualitative case study of ImpactCo, an ImS venture based in India.

\section{SPACES AND PLACES}

Sociology of space is a branch of sociology that broadly concerns itself with understanding the "social and material constitution of space". Space here, for example, can refer to either the 
"physical space" or land, building and so on and the "social space”, or space for social relations and interactions. However, in extant literature, the distinctions are not so straightforward. A number of eminent classical sociologists (see Urry (2000) for a brief review), human geographers (Lobao, 1996) as well as contemporary sociologists (Giddens, 1990; Lefebvre, 1974; Gieryn, 2000; Bourdieu, 1996) have tried to outline a sociology of space and place. These scholars, concerned with examining the spatial dimension of societies, have theorized space and place in contrasting manners (D’Mello and Sahay, 2007). However, in these theorizations, the analytical boundaries between space and place overlap. For example, Bourdieu (1996) classifies space as physical and social space. He refers to physical space as reified social space, illustrating the difference with the example of neighborhoods and communities - both, which are social formations that come into being as a result of prolonged social interaction. Similarly, scholars in the human geography discipline refer to place as a "particularistic dimension" of space and social space as having a "relational dimension” (Lobao, 1996). Gieryn (2000), outlining a "sociology of place", on the other hand, offers a clearer distinction between space and place. He contends that place has a geographic location, material form and assigned meaning and value. On the other hand he identifies space as a set of "abstract geometries detached from material form and cultural interpretation” (p.465). D’Mello and Sahay (2007) and Sahay, Nicholson and Krishna (2003) too make similar distinctions. However, on the other hand Lefebvre (1974) contends that space is not passive geometry and that "space is produced and reproduced and represents sites of struggle” (Urry, 2000, p.11).

From this very brief review we get a sense that there may be an issue of semantics, or of course the meaning of space and place as interpreted by scholars may be evolving (see D’Mello and Sahay, 2007). Consequently, a number of ontological questions arise: Are spaces and places different? Do spaces constitute spaces? Are they a continuum? When do spaces become places and vice-versa? For the purpose of this paper, Simmel's treatment of 
space and place has been adopted. He suggests that space is an "ineffectual form” brought to life by the "filling of space" through social interactions (Frisby and Featherstone, 1997). “Simmel's” space seems conceptually similar to what Bourdieu (1996) refers to as "social space”. Further, the qualities of social space, i.e. having meaning and value, are relational, and have a geographic location, all seem very similar to scholar’s who theorize "place”. In this paper we therefore use the terms space, social space and place interchangeably.

Simmel in his essay, “The sociology of space” (Frisby and Featherstone, 1997) explores the spatial dimension of society. He suggests that social interactions have a spatial form and these interactions turn space into something meaningful. Simmel's epistemology suggests that space does not wholly determine social interactions and neither does it imply that the construction of space is purely a social constructionist process (Lechner, 1991; Frisby and Featherstone, 1997). Meaning, space retains a character of its own, but at the same time influences how social groups experience and produce their own space (Gieryn, 2000). Simmel outlines several fundamental qualities of spatial form upon which the structuring of communal life relies. The first of these qualities is what he terms the exclusivity of space, or the exclusivity with which social groups treat space. He suggests that spatial forms may vary in the extent to which their existence depends upon exclusive occupation of space. For example, no two nation-states can occupy the same space; they require exclusive occupation of space. The second fundamental quality of space which Simmel examines is the boundaries created by space. Boundaries, Simmel suggests is a sociological fact with spatial consequences and not the other way around (Frisby and Featherstone, 1997, p.143). Meaning, while geographical boundaries may exist, it is the psychological forces of the individuals within the space which determine its boundary. Boundaries may therefore get stretched beyond their geographical confines (Massey, 1984). The third aspect of space which affects social formations is what Simmel refers to as the ability of space to fix their contents. Again here, the extent to which the objects, groups and individuals of the social formation require 
fixity affects Simmel suggests that space has the capacity to provide stability to social interactions especially through the objects which occupy and remain fixed in space. For instance, places of worship such as a church, a temple, a mosque or a synagogue, which are spatially immovable, serve as pivot points in space around which social interactions take shape, eventually providing stability to a social group. The last aspect of space which we will discuss is the sensory proximity which space affords to social interactions. Social formations, depending on their configuration, may require its contents, i.e. social groups and other associated objects to be either within close proximity. This proximity has further consequences for the unity of the social group and its formation. As Simmel notes, conversational proximity creates a much more individual relationship as opposed to only visual proximity. Overall, Simmel's analysis is useful to understand how social interactions are structured through space.

\section{IMPACT SOURCING, SPACES AND PLACES}

The practice of hiring and training marginalized individuals to provide digitally-enabled services is a relatively new phenomenon in IT-BPO. There is a growing call for research to explore and understand this nascent phenomenon (Carmel, Lacity and Doty, 2013; Heeks, 2013). One important area for research is developing an understanding of the nature of "impact" of ImS ventures as the phenomenon is popularized around the idea that it has the potential to create positive impact. In line with this call, a small, but growing number of studies have analysed how ImS employees and the larger community are impacted by ImS activity. For example, Heeks and Arun (2010) analysed a government-driven ImS model that aimed to improve livelihood opportunities of marginalized groups in the state of Kerala, India. More recently, studies have noted how employment in ImS ventures has resulted in positive impacts such as improved cognitive abilities, development of self-esteem and confidence, and improved capabilities (Madon and Sharanappa, 2013; Malik, Nicholson and 
Morgan, 2013). The aforementioned studies focussed on ImS ventures based in rural India. Lacity, Rottman and Carmel (2014) studied an ImS model at work in a penitentiary system in the United States of America. Here, the beneficiaries were prison inmates trained and employed to work on IT-enabled projects. This study too reports increased self-efficacy and self-esteem among the workers.

Although extant literature in ImS does not specifically examine how space and place impacts ImS employees, there are some studies in the broader IT-BPO literature that have used space and place as lenses to explore phenomenon. Sahay, Nicholson and Krishna (2003)'s analysis of the Globtel-MCI case revealed a dialectical tension between space and place. The case illustrates Globtel's treatment of software development as situated in a global space coming into tension with the viewpoints of individuals who strive to retain the localness in their workplace. In another study, D’Mello and Sahay (2007) employ the place lens to understand mobilities of individuals working in global software organizations and their interaction with place and space. Similar to Sahay, Nicholson and Krishna (2003), D’Mello and Sahay (2007) suggest that despite the globalized nature of work individuals constantly strive to remain rooted in a place they identify with.

Indeed as Gieryn (2000) notes, place affects individuals and social groups in important ways. Place "stabilizes and gives durability to social and structural categories, differences and hierarchies; arranges patterns of face-to-face interactions that constitute network formation and collective-action; embodies and secures otherwise intangible cultural norms, identities and memories” (p. 473). In this paper it is argued that social space or place can influence the process of sociation among individuals, through which they experience social and psychological changes. Here, ImS ventures can be viewed as producers of physical space, which act as spaces of change in predominantly traditional communities. The hitherto nonexistent physical spaces, to use Oldenburg's (1989) idea, serve as a hybrid of the "second" 
(place of work) and “third” (place of play) space. The newly created space, we argue, opens up marginalized individuals to new experiences, exploration and learning. Individuals occupying these physical spaces engender new "social spaces” (Bourdieu, 1990) driven by their "new" habitus (created by the ImS venture). Here, empowerment of marginalized individuals can be viewed as resulting from the set of capabilities or strategies they develop to negotiate the tensions posed by the conflict between the "old" (traditional, orthodox) and the new (liberal, egalitarian) habitus.

\section{RESEARCH METHODS}

For an in-depth understanding of the lived experience of the employees of ImpactCo we adopted an interpretive case-study approach (Walsham, 1995). Researchers adopting the case-study approach spend considerable time in the field interacting with their informants via semi-structured and open-ended interviews, and being observers of organizational life as participants or non-participants (Yin, 1994).

\subsection{Research site}

ImpactCo, an ImS venture, is a for-profit social enterprise based in the lower Himalayan regions of North India. The venture was founded in 2009 by two Indian entrepreneurs with considerable IT-BPO experience at top management level. ImpactCo was started with the intention of providing employment opportunities to marginalized youth in the rural Himalayan region. ImpactCo hires and trains individuals from local villages on IT and managerial skills. After completing an initial training period, the recruits are deployed to work on projects sourced from domestic and international clients.

ImpactCo opened its first centre in the year 2009 employing close to fifty individuals from the local and nearby villages. As of 2014, ImpactCo has established six centres employing close to 400 individuals. The centres are located in different villages, all within a radius of 50 
kilometres. On an average there are about 300 households in each village. In this paper the six villages are collectively referred to as "the community". The major source of livelihood for the people in the community is agriculture and horticulture. During our fieldwork, we observed that the community members functioned strictly in line with their traditions, and were fairly orthodox in their world views.

\subsection{Data sources}

Our research is primarily informed by empirical material collected over a six-month fieldwork (from March 2013 to August 2013) of ImpactCo. We collected data through openended interviews and non-participant observations. Table 1 below gives an overview of our informants. Overall, we interacted with 25 employees of ImpactCo across their five centers. We also had conversations with members of the community during our fieldwork.

\begin{tabular}{|l|c|c|c|c|c|c|}
\hline & Centre 1 & Centre 2 & Centre 3 & Centre 4 & Centre 5 & Total \\
\hline Male & 2 & 2 & 3 & 1 & 0 & 8 \\
\hline Female & 3 & 5 & 7 & 2 & 1 & 17 \\
\hline Total & 5 & 7 & 10 & 3 & 1 & $\mathbf{2 5}$ \\
\hline
\end{tabular}

Table 1. Overview of informants

The first author conducted unstructured and open-ended interviews with ImpactCo employees and community members. The interviews lasted anywhere between 15 minutes to more than two hours. Interviews with ImpactCo employees and community members were conducted in Hindi and sometimes in English. These interviews were recorded, transcribed and translated (when needed). Detailed notes about conversations with informants were made at the end of each day of fieldwork. 


\subsection{Data analysis}

We conducted data analysis over several stages. In the first stage a database was built comprising of empirical material relating to the beneficiaries' experience of working in ImpactCo. In the second stage a detailed analysis of this database was conducted to broadly understand (1) how social space influenced social interactions and (2) how beneficiaries constructed social space. In the third stage, thematic data analysis techniques (Miles and Huberman, 1994) were used to identify initial codes drawing on Simmel's categorization of fundamental qualities of spatial forms. The fourth stage involved examining the codes to look for patterns and identifying themes. Overall, four different themes and a few subthemes were identified and grouped together. They were: (1) beneficiaries' perception of the exclusivity with which the community and ImpactCo viewed their space (2) how beneficiaries managed boundaries created by space (3) how beneficiaries navigated the challenges of working in a new environment and finally (4) how beneficiaries experienced their new found independence at ImpactCo workplace. We revisited our empirical material and travelled back and forth iteratively from the data to the literature on sociology of spaces until there was a reasonable fit between data and theory and no further iterations seemed valuable.

\section{ANALYSIS}

In this section we present our interpretation of the lived experience of our informants. Drawing on Simmel's work, we discuss the everyday struggles of ImpactCo employees as they navigate the old (the community) and the new (ImpactCo) space.

\subsection{Us and them: exclusive spaces}

We understood through our informants that patriarchy and orthodoxy held sway in the community where ImpactCo had its operations. For the youth of the community, obeying their elders was of paramount importance, and their freedoms were largely regulated their 
parents. There were restrictions around how men and women (especially those who were not known to each other) interacted in public life and there were consequences for transgressors. Community members upheld and followed inherited traditional values, beliefs and customs. The norms and values guiding organizational life at ImpactCo was in stark contrast to that of the community. ImpactCo espoused liberal principles and ideas such as gender equality and freedom of speech. The ImpactCo space was therefore viewed by the community members as something foreign and alien - essentially the polar opposite to the culture of the community. The two mutually exclusive spaces had several implications for the employees of ImpactCo.

As the informants of the study noted, they had a feeling that they were being constantly watched over by other members of the community for any “deviant” behaviour. Again, while working within the confines of ImpactCo they were mindful of how they fit in with the culture of the organization. ImpactCo employees therefore confronted challenges as they navigated between their work and non-work contexts. In the sections that follow, we elaborate on the challenges faced by ImpactCo employees and the strategies they devised to prevail over their predicament.

\subsection{Managing across cultures: boundaries created by space}

The notion of exclusive space also implies the presence of boundaries. These boundaries however are not just physical ones. In fact, as Simmel suggests boundaries are sociological facts with spatial consequences (Frisby and Featherstone, 1997, p.143). Therefore while physical boundaries may exist, it is the psychological nature of boundaries that more acutely define social interactions. Here, boundaries serve the dual function of closing space off against the surrounding world and holding the space together. Meaning, boundaries have the capacity to hold and unite social groups at the same time differentiate from other social groups or formations. Since, the community space and ImpactCo space were in stark contrast, 
the personal mobility across cultures and spaces posed challenges for employees who occupied both these space.

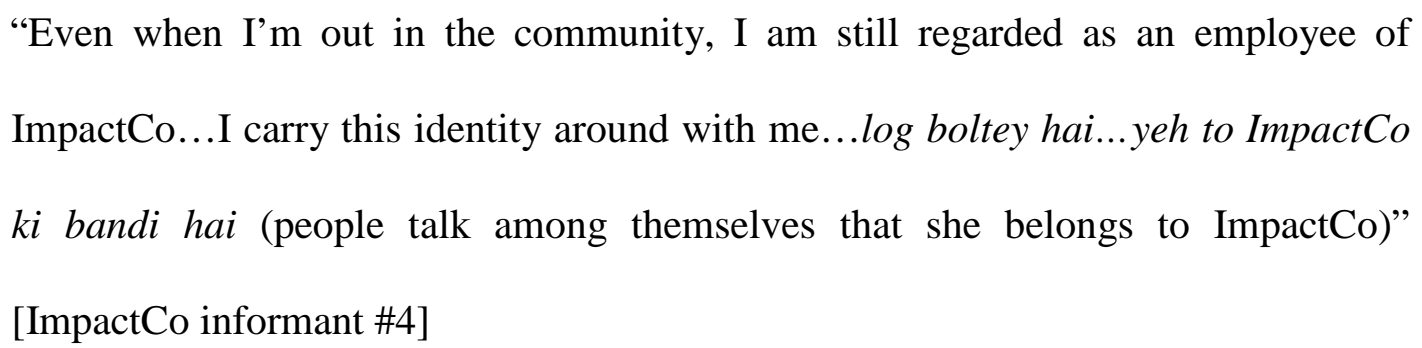

Employees who crossed over from the community space to ImpactCo space faced the challenge of managing different social and cultural expectations. There were mainly two boundaries that employees managed (1) the ImpactCo-Community boundary and (2) the ImpactCo-Home boundary. Broadly, there were two strategies that employees adopted to manage these boundaries.

In the first instance, individuals adopted what we call a chameleon strategy. Meaning, they embraced the new culture espoused within the ImpactCo space while at work, however, when they were in the community space, they embraced the dominant values prescribed by the community.

\footnotetext{
"When I am in the office I am like how everyone else is...but when I am in the community I am how I am expected to be...pahaad ke hisaab se rahna chahiye (should live according to the customs of the hills; as opposed to the "plains", in this context the plains refer to the cities) [ImpactCo informant]
}

In the second instance, individuals carried the new culture espoused within the ImpactCo space back to the community and their homes.

In the beginning I used to get asked many questions... Why are you hanging out with those boys? Why do you laugh and talk so loudly while in the open? But over time, I introduced my new friends to my parents. I also made it a point to bring my parents 
to the workplace so that they get familiar with where I work and who I interact with...” [ImpactCo informant]

These two strategies of managing the boundaries can be broadly identified with the process of acculturation of individuals. Acculturation is "a dynamic and multidimensional process of adaptation that occurs when distinct cultures come into sustained contact”. The first strategy can be viewed as an integration strategy. Integration is defined by Berry (2003) as "when a person shows an interest in maintaining the original culture and in learning and participating in the other culture(s).” Employees did show keen interest to learn about the new culture and imbibe new cultural practices; however at the same time they were eager to maintain and "perform” their older culture in the community. Similarly, the second strategy that employees adopted can be viewed as an assimilation strategy. Assimilation is when an individual wishes to diminish or decrease the significance of the culture of origin and desires to identify and interact primarily with the other culture. Here, employees were eager to maintain their newly acquired cultural tools even in the community space.

In the process of managing these boundaries, employees learned to cope with different cultural expectations imposed by different spaces. In the next two sections we discuss how the ImpactCo space shaped social interactions among the employees.

\subsection{New threats, new technology \& practices: space creating proximity}

Another characteristic of space is its ability to provide sensory proximity between people who occupy the same space. Furthermore, each social form has its own requirement of proximity. Meaning, some social forms may require individuals to be in close proximity, while others can do with individuals being dispersed. In the ImpactCo space, there was an obvious need for people to congregate in a single space and spend a lot of time working together and socializing because of the nature of the work and workplace. The degree of contact and the amount of time people spent talking to each other was also high because of how work was 
structured: teams of people working on the same task. This characteristic of the ImpactCo space had some implications.

\subsubsection{Space and threats}

Most individuals employed by ImpactCo were fresh out of high school or college with little or no experience of working in a professional environment. Additionally, working in ImpactCo was an altogether different cultural experience. Rarely had men and women shared the same space and interacted with people who they were not familiar with or not related to. As a female employee of ImpactCo recounted her first week in the office:

During the first day of our orientation all the boys ran to one section of the class and us girls ran to the other...there was so much hesitation to talk, to make eye contact...we were so not used to it...I was especially not so used to being around so many boys I did not know. [ImpactCo informant]

It was not just working with the "other” gender that made people uncomfortable. The fact that they were in an environment where professionalism was expected and an unfamiliar workplace full of IT artefacts flustered many.

I was very afraid initially, darte hue hi kaam kar raha tha (I lived and worked with fear)...I was not at all familiar with working in an office like this...I was a salesman in a Kashmiri handicrafts shop earlier. [ImpactCo informant]

I finished school and I was all ready to get married. But, the marriage got cancelled so I wanted to work. To be honest, I was so frightened, I came from another village and I did not know anyone here. I wasn’t sure if I would be able to do all the processes...this was completely new to me. [ImpactCo informant] 
Being in an unfamiliar environment, working and socializing with the other gender were turning out to be an overwhelming experience for most of our informants. Over time they had found a way to deal with their unsettling environment.

I thrive in an environment where I know other people around me...where I do not feel fear...but these were people I did not know and I had never been in this situation earlier. I slowly opened myself up to others...I found it difficult initially because I’ve never been an outgoing person... [ImpactCo informant]

Earlier I never used to interact with men. But here we have a chance to understand each other as men and women. [ImpactCo informant]

During our fieldwork the social bonds among the employees were conspicuously high. Recounting how she felt when she had to move from one ImpactCo center to another, an employee noted:

We are like a family here. When I was moved from one center to another, I cried so much! I did not even cry that much when I left home the first time. My colleagues are more than just my colleagues...they are my family, my brothers and sisters. They give me confidence and encourage me to do better. [ImpactCo informant]

Employees developed deep social bonds to the extent that they considered other members of their team and group as one of their own member of family. These bonds went beyond the realm of what is typically branded as "collegiality" in a workplace. This type of social formation closely relates to the idea of a fictive kinship or pseudo-families (Carsten, 1995). These are strong social bonds not based on blood but contextual factors. Here the basis for the existence of a fictive kinship was to help each other thrive in an unfamiliar environment.

\subsubsection{Space, new technology \& practices}


The proximity afforded by the ImpactCo space had some "enabling" implications. Most employees were technology illiterates. Very few had any experience of working word processors, spreadsheets and attending to basic administrative tasks using a personal computer. In addition, very few had any professional experience and were not familiar with the typical practices followed in an organization. Recalling his initial week at the induction, an ImpactCo informant noted:

They sat us down in front of systems. Each one of us was given a task to complete. We had to type in a paragraph of text into Microsoft Word and save the document using a filename of our choice. I typed in "Kuch" (which literally translates to “something”)...pressed some button and I lost sight of the document. I panicked and tried various things but the document refused to re-appear. When my instructor noticed my panic he asked me what had happened. I said I lost "something”! I couldn’t even articulate what exactly I had lost. [ImpactCo informant]

Not only was working with new technology intimidating. New entrants also had to quickly learn the norms and values espoused by ImpactCo and act their part to show that they belong to the group. Learning technical skills as well as the more symbolic elements of conducting oneself in the workplace setting was turning out to be overwhelming.

I was not used to shaking hands with men, let alone giving them a hug! I found it all very strange. I felt like I had entered a new world altogether. All of us in the new batch used to wonder "kaise baatey kar rahe hain" (look at how they are talking and behaving!). [ImpactCo informant]

There was a pressure to fit in to this newfound culture - new vocabulary to pick up, new mannerisms to learn. Coming from a distant reality, the scenarios they were encountering at 
work were proving to be a cultural shock. Our informants recognized that they had to very quickly pick up cues and broaden their "cultural toolkits".

During the first two weeks of induction I observed a lot...how people spoke, how they interacted with their Team Leads and also during our morning huddles - how people discussed issues. Also how they greeted each other hugged and shook hands. Then I tried to copy them as much as I could! [ImpactCo informant]

I am a TL now... all thanks to Rahul sir, he helped me a lot and I learnt a lot from him. I used to see how he handled team meetings and problems with staff. I still have a long way to go...I look up to him for guidance and inspiration. [ImpactCo informant]

Employees mimicked practices of other more experienced employees. In this way they were ensuring compliance with ImpactCo’s cultural norms. Essentially, our informants were creating what is referred to in literature as "provisional selves” (Ibarra, 1998). This strategy allowed individuals to try different self-presentations, mimicking norms and behaviour which they believed were crucial in their workplace.

\subsection{Stability, individuality \& dignity: space and its capacity of “fixing”}

Prior to ImpactCo, the youth of the community, especially for the men and women, did not have a legitimate space to interact and socialize. Most gatherings were around the time of festivals and predominantly with kith and kin. Mingling of a man and a woman not known to each other was widely a social taboo with the intentions of the interactions being watched and scrutinized by community elders.

Going out was not allowed - not even to my relatives place...my parents used to be apprehensive. Forget meeting strangers! [ImpactCo informant] 
ImpactCo created a hitherto non-existent "stable" space where the youth of the community could meet and socialize.

Earlier we never had opportunities to meet new people and learn new things. Yes, there were fairs in villages and in towns...but that was only once a year...and during festivals too we got to meet...there was no other place... [ImpactCo informant]

Space, as Simmel suggests has this quality of “fixing” contents. Consequently, social interactions, which revolve around these contents, also become "fixed". By becoming the focal point of interaction, ImpactCo space localized and crystalized the community life of ImpactCo employees. The immovable nature of the contents, i.e. the workplaces of ImpactCo, provided stability to these social interactions.

ImpactCo thus served as a pivotal point for social interactions and engendered a sense of community. More importantly, this also awakened a sense of belonging among its employees.

When I wear the ID tag around my neck I feel proud that I am part of ImpactCo...that I am part of something big... I am blessed to be associated with this organization. [ImpactCo informant]

Alongside instilling belongingness, ImpactCo space also gave its employees a sense of individuality and dignity.

I eagerly look forward to coming here every day. I feel that I am somebody here. Back at home, as a girl, I do not have much freedom to express myself... and the whole day goes away doing odd tasks... This is a place where I can try to be myself. [ImpactCo informant] 
The layout of the work center was typical of any urban BPO center. Each employee was assigned a particular personal computer on which they had to work on. Employees adorned their personal space on their desks with images of their favorite God or other memorabilia. Motivational posters that they had designed during their induction adorned the walls. There was a communal space within their work centre where they hung out during tea breaks to engage in banter.

It's a different feeling, having your own system and your chair. We cannot afford these at home... It's a place I can call my own... [ImpactCo informant]

\section{DISCUSSION}

Overall, the analysis revealed that individuals experienced social and psychological changes while navigating the complexities posed by a) the conflict between the community and the ImpactCo space and b) the new social and cultural environment of ImpactCo space. Evidently, the mutually exclusive nature of ImpactCo space and the community space created an environment ripe for new learning and experiences for ImpactCo employees. The spatial divisions between the two spaces further coerced ImpactCo employees to manage social and cultural boundaries.

\subsection{Theoretical implications}

The paper answers to recent calls for research on the ImS phenomenon (Carmel, Lacity and Doty, 2013; Madon and Sharanappa, 2013; Malik, Nicholson and Morgan, 2013). Specifically, the paper contributes to the emerging discourse on the impact of ImS ventures. It explores the lived experience of ImS employees and tries to understand how they navigate the challenges brought about by the new ImS workplace. The key contribution of the paper is its exploration of how ImS employees manage their transition into ImS workplaces from their 
traditional communities. The study identified acculturation strategies of assimilation and integration used by ImS employees to manage boundaries between work and non-work spaces. ImS employees also developed strategies such as forming fictive kinships and experimenting with provisional selves to cope with challenges arising within the $\operatorname{ImS}$ workplace. More broadly, the study highlighted the transformative role of ImS workplaces. The study also implicitly demonstrates the multifaceted nature of "impact”. Based on our findings we would argue that there are broadly two kinds of impacts: intended and experienced impacts. Here, intended impact refers to the organizational efforts of the ImS venture to fulfil their organizational mission - which is invariably - providing sustainable livelihood opportunities to marginalised communities. In this paper, we have tried to understand and explore the latter type of impact, i.e. experienced impact, from the point of view of ImS employees. Here, the development of various strategies to navigate the complex social and cultural challenges can be viewed as impacting the personal development of ImS employees.

\subsection{Practical implications}

Primarily, our paper highlights the need for ImS ventures to be cognizant of the complex nature of impact. A number of ImS ventures are being funded by so called "social venture" capital funds. Invariably such funds also come with the requirement that organizations measure and document their "social impact" and further rounds of funding may be contingent upon organizations demonstrating that they have created an impact. However, most organizations rarely go beyond documenting “economic impact”. There can be of course a number of reasons behind this. Organizations may not have the budget and resources to evaluate and monitor the impact they are creating. Regardless, it may be of immense benefit to the organizations to understand how their employees learn to work in a new environment. This kind of understanding may serve an important practical purpose. Organizations can 
devise mechanisms to formalize and incorporate some of these learning methods to benefit more employees.

\subsection{Limitations, future research and conclusions}

Our study is not without its limitations. This study is based on a single case and therefore can be generalized only to other cases in similar contexts. The learning and coping strategies which we elicited were embedded in a particular context and the strategies of ImS employees may vary with the context. We encourage other researchers to study how ImS employees cope with the challenges of new space in different contexts. We would also encourage researchers to study coping strategies of other populations of marginalized individuals that ImS ventures are impacting, such as persons with disabilities, low income urban youth and so on.

As the ImS model grows in popularity, scholars have begun to study different aspects of the model. The study adds to this growing stream of literature on ImS models. Specifically it explores the lived experience of marginalized individuals employed in an ImS venture and examines their everyday challenges of coping with the challenges of living and working in two distinct spaces. The findings of the study have implications for ImS companies and other organizations working with marginalized individuals. As ImS companies grow and expand in the global south, the study encourages practitioners and academics to understand how marginalized individuals are experiencing and managing dialectical tensions.

\section{REFERENCES}

Accenture. (2012) Exploring the value proposition for Impact Sourcing: A Buyer's perspective. Available at: http://www.accenture.com/SiteCollectionDocuments /PDF/Accenture-Exploring-Value-Proposition-Impact-Sourcing.pdf (Accessed: 14 September 2014). 
Barnes, A. (2007) “The construction of control: the physical environment and the development of resistance and accommodation within call centres”, New Technology, Work and Employment, 22(3), pp. 246-259.

Berry, J. W. (2003) 'Conceptual approaches to acculturation', in K. M. Chun, P. Balls Organista, and G. Marín (eds.), Acculturation: Advances in theory, measurement, and applied research. Washington, DC: American Psychological Association, pp. 17-37.

Bourdieu, P. (1990) In Other Words: Essays towards a Reflexive Sociology. Stanford, CA: Stanford University Press.

Bourdieu, P. (1996) “Vilhelm Aubert memorial lecture: Physical Space, Social Space and Habitus”. Available at:

http://archives.library.illinois.edu/erec/University\%20Archives/2401001/Production_website/ pages/StewardingExcellence/Physical\%20Space,\%20Social\%20Space\%20and\%20Habitus.pd f (Accessed: 10 January, 2015).

Carmel, E., Lacity, M. and Doty, A. (2013) The Impact of Impact Sourcing: Framing a Research Agenda. $4^{\text {th }}$ International Conference on the Outsourcing of Information Services, Mannheim, Germany.

Carsten, J. (1995) “The substance of kinship and the heat of the hearth: feeding, personhood, and relatedness among Malays in Pulau Langkawi”, American Ethnologist, 22 (2), pp. 223241.

D’Mello, M. and Sahay, S. (2007) ““'I am kind of a nomad where I have to go places and places"'... Understanding mobility, place and identity in global software work from India”, Information \& Organization, 17 (3), pp. 162-192.

Frisby, D and Featherstone, M. (1997). Simmel on Culture. London: Sage. 
Giddens, A. (1990) The consequences of modernity. Cambridge: Polity Press.

Gieryn, T.F. (2000) “A space for place in sociology”, Annual Review of Sociology, 26, pp. 463-496.

Heeks, R. and Arun, S. (2010) "Social outsourcing as a development tool: the impact of outsourcing IT services to women's social enterprises in Kerala”, Journal of International Development, 22 (4), pp. 441-454.

Hirst, A. (2011) "Settlers, vagrants and mutual indifference: unintended consequences of hotdesking”, Journal of Organizational Change Management, 24 (6), pp. 767-788.

Ibarra, H. (1999) "Provisional selves: Experimenting with image and identity in professional adaptation”, Administrative Science Quarterly, 44 (4), pp. 764-791.

Klein, H. K. and Myers, M.D. (1999) “A Set of Principles for Conducting and Evaluating Interpretive Field Studies in Information Systems”, MIS Quarterly, Special Issue on Intensive Research, 23 (1), pp. 67-93.

Lacity, M., Rottman, J.W. and Carmel, E. (2014) "Impact Sourcing: Employing Prison Inmates to Perform Digitally enabled Business Services”, Communications of the Association for Information Systems, 34 (51), pp. 913-932.

Lacity, M.C., Rottman, J. and Khan, S. (2010) "Field of dreams: building IT capabilities in rural America”, Strategic Outsourcing: An international journal, 3 (3), pp. 169-191.

Lechner, F.J. (1991) "Simmel on Social Space”, Theory, Culture and Society, 8 (3), pp. 195201.

Lefebvre, H. (1974) The production of space. Translated from french by D.N.Smith Oxford: Blackwell. 
Lobao, L. (1996) “A Sociology of the Periphery Versus a Peripheral Sociology: Rural Sociology and the Dimension of Space”, Rural Sociology, 61 (1), 77-102.

Madon, S. and Sharanappa, S. (2013) "Social IT sourcing and development: Theorizing the linkage”, Information Systems Journal, 23 (5), pp. 381-399.

Malik, F., Nicholson, B. and Morgan, S. (2013) “Assessing the social development potential of impact sourcing”, Proceedings of the $6^{\text {th }}$ Annual Conference SIG GlobDev, Milan, Italy.

Massey, D. (1998) Space, place and gender. Cambridge, UK: Polity Press.

Miles, M.B. (1994) Qualitative data analysis: An expanded sourcebook. Thousand Oaks, California: Sage.

Oldenburg, R. (1989) The Great Good Place: Cafes, Coffee Shops, Community Centers, Beauty Parlors, General Stores, Bars, Hangouts, and How They Get You Through the Day. New York: Paragon House.

Rockefeller. (2013) Digital Jobs: Defined. Available at: http://www.rockefellerfoundation.org/our-work/current-work/digital-jobs-africa/digital-jobsdefined (Accessed: 1 November 2013).

Sandeep, M.S. and Ravishankar, M.N. (2013) The other India, Professional Outsourcing, 13, pp. 14-20.

Sandeep, M.S., Ravishankar, M.N. and Hislop, D. (2013) “The Establishment of social IT sourcing organizations: An impression management perspective”, Proceedings of the International Conference on Information Systems, Milan, Italy.

Sahay, S., Nicholson, B., and Krishna, S. (2003) Global IT outsourcing: Software development across borders. Cambridge, UK: Cambridge University Press. 
Urry, J. (2004) 'The sociology of space and place', in Blau, J.R. (ed), The Blackwell companion to sociology. Oxford: Blackwell, pp 3-15.

Walsham, G. (1995) “The emergence of interpretivism in IS research”, Information Systems Research, 6(4), 376-394.

Yin, R. K. (1994) Case study research: Design and methods. Thousand Oaks, CA: Sage. 